\title{
EBW Power Deposition and Current Drive in WEGA - Comparison of Simulation with Experiment
}

\author{
Josef Preinhaelter ${ }^{1}$, Heinrich P. Laqua ${ }^{2}$, Jakub Urban ${ }^{1}$, Linda \\ Vahala $^{3}$ and George Vahala ${ }^{4}$ \\ ${ }^{1}$ Institute of Plasma Physics AS CR, v.v.i., Association EURATOM/IPP.CR, \\ Prague, Czech Republic \\ ${ }^{2}$ Max-Planck-Institut für Plasmaphysik, EURATOM Ass. Greifswald, Germany \\ ${ }^{3}$ Old Dominion University, Norfolk, VA 23529, USA \\ ${ }^{4}$ College of William \& Mary, Williamsburg, VA 23185, USA \\ E-mail: preinh@ipp.cas.cz
}

\begin{abstract}
Detailed computational studies of electrostatic electron Bernstein waves (EBW) propagation in the WEGA stellarator are performed and compared to experimental results. Using the WEGA antenna, the two O-/X-mode radiation lobes are modeled by sets of rays whose intensities are proportional to the measured radiation pattern. After projecting these rays onto the plasma periphery, the O-X-EBW mode conversion efficiency around the upper hybrid resonance is determined from a full wave adaptive mesh solver of the cold plasma equations. From the roots of the electrostatic EBW dispersion relation, ray tracing is performed to determine the power absorption on the first or second cyclotron harmonic as well as current drive assuming the FischBoozer mechanism. Good agreement is achieved between our EBW simulations on specific WEGA equilibria and the experimental results from the antenna launch of 2.45 $\mathrm{GHz}$ waves. The experimentally observed off-axis power deposition and the outward shift dependence of the absorption maxima on increasing magnetic field can only be explained by the existence of a hot electron component in the WEGA plasma. It is this hot electron component that permits wave absorption at the second harmonic near the plasma boundary. Moreover, the simulations not only reproduce the current density reversal at the plasma centre for low magnetic fields but also the destruction of this current density reversal for larger magnetic fields.
\end{abstract}

PACS numbers: $52.35 . \mathrm{Hr}, 52.25 . \mathrm{Os}, 52.55 . \mathrm{Hc}$

\section{Introduction}

Electron Bernstein wave research is of major importance for magnetic fusion devices operating in high-density regimes - in particular, for stellarators and spherical tokamaks. In these overdense plasmas, the standard ordinary $(\mathrm{O})$ and extraordinary $(\mathrm{X})$ modes are cutoff when the electron plasma frequency becomes much larger that the electron cyclotron frequency. In this regime, only the electrostatic electron Bernstein wave 
(EBW) can propagate. EBW physics is more complicated than that for the electromagnetic O- and X-modes, primarily due to their electrostatic nature. Since electrostatic EBW waves have a very strong interaction with the plasma, their dispersive properties are quite difficult to predict. Moreover, since electrostatic waves cannot propagate in a vacuum or in a rarefied plasma, these (internal) EBW waves must be mode converted around the upper hybrid resonance layer from the O- or X-mode.

In this paper, we report on both simulation and experimental studies of EBW heating and current drive in the overdense WEGA stellarator - a stellarator that has a fundamental program in place to study EBW physics.

In Sec. 2.1 we briefly describe the plasma parameters of the WEGA stellarator and its antenna system, while in Sec. 2.2 we present the major experimental WEGA results on power deposition and current drive profiles for $2.45 \mathrm{GHz}$ waves.

In Sec. 3 we summarize the theoretical underpinnings of our simulation code, paying particular attention to the effect of collisions with neutrals as well as inelastic collisions with ions. These effects are very important when WEGA is run with an Argon plasma. The dispersion relation for ray tracing is described together with the determination of current density and power deposition.

In Sec. 4 we present simulation results obtained from our AMR code (Antenna, Mode conversion, Ray tracing), a code initially developed for studying EBW physics on spherical tokamaks (MAST and NSTX). The O-X-EBW mode conversion efficiency for obliquely incident $2.45 \mathrm{GHz}$ waves is considered in Sec. 4.1. The ray trajectories and the development of $N_{\|}$are discussed in Secs. 4.2 and 4.3. In Sec. 4.4 we investigate the effects of the hot plasma component content on the wave propagation and damping, while in Sec. 4.5 we present simulation results on the magnetic field dependence of the power deposition and current density profiles. Finally, in Sec. 4.6 we show that the current reversal is due to a very large increase in $N_{\|}$along the ray trajectory with central power absorption on the hot electrons at the second cyclotron harmonic.

\section{Experimental setup and measurements}

\subsection{The WEGA stellarator}

WEGA is a medium-sized classical $\ell=2, m=5$ stellarator with major radius $72 \mathrm{~cm}$ and maximum minor plasma radius of $13.5 \mathrm{~cm}$ [1]. It consists of five identical sections, each spanning $72^{\circ}$ in the toroidal direction. Although WEGA is equipped with an iron OH-transformer with a capability of $440 \mathrm{mVs}$, in the EBW studies it was only used to measure the toroidal plasma current. The magnetic field configuration is deduced from the VMEC code [2] and processed by a library. This provides all the magnetic field data: $s, \mathbf{B}$, and $\mathrm{d} \mathbf{B} / \mathrm{d} \mathbf{r}$ at any spatial point (here $s$ is the normalized toroidal magnetic flux: $s=0$ on the plasma axis and $s=1$ on the last closed flux surface (LCFS)). The WEGA equilibrium is chiefly controlled by external coil currents although there is a minor correction due to the plasma pressure gradient. Thus the magnitude of the central 
magnetic field $B_{0}$, together with the plasma density and temperature profiles, can be modified without considerably changing the equilibrium. It is convenient to express $B_{0}$ in units of the resonant (electron) magnetic field at frequency $f: B_{\mathrm{ce}}=2 \pi f m_{\mathrm{e}} / e$. For the WEGA experiments, $f=2.45 \mathrm{GHz}$ so that $B_{\mathrm{ce}} \cong 0.0875 \mathrm{~T}$. For the WEGA experiments we shall examine, the typical central magnetic field values lie in the range $0.6 B_{\text {ce }}<B_{0}<0.9 B_{\text {ce }}$ and the rotational transform $\iota=0.2$.

The plasma was created using $26 \mathrm{~kW}$ of ECRH at $2.45 \mathrm{GHz}$. A standard WEGA steady state discharge (in either hydrogen, helium or argon plasma [1]) lasts up to 30 s with optimal heating being achieved by a $\varnothing=83 \mathrm{~mm}$ waveguide antenna with two slots in the sidewall (slot length $10 \mathrm{~cm}$, angular width $64^{\circ}$ ). At $2.45 \mathrm{GHz}$, only the TE11 mode can propagate, leading to an HF emission which is linearly polarized in the horizontal plane. The antenna radiation pattern has two lobes with launch angles $\theta= \pm 60^{\circ}$. In our simulations we model the measured double lobe radiated power by two beams with fixed divergence having slightly deformed Gaussian profiles.

\subsection{ECRH heating and current drive}

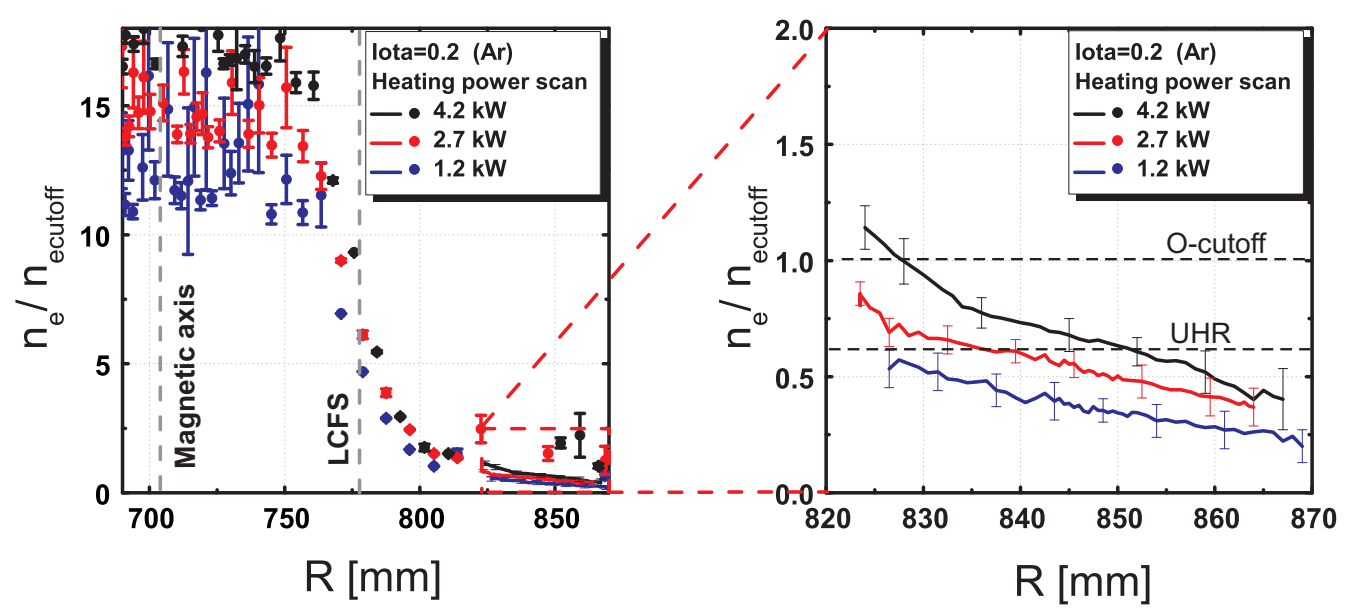

Figure 1. Left: Plasma electron density calculated from the ion saturation current for the $-90 \mathrm{~V}$ applied voltage assuming $T_{\mathrm{e}} \cong 5 \mathrm{eV}$. $T_{\mathrm{e}}$ is determined from the probe current voltage characteristic and does not vary much over the plasma radius - for details see [4]. $B_{0}=0.65 B_{\text {ce }}$. Right: Electron density in the vicinity of the ECRH antenna outside the last closed flux surface together with the mode conversion layers for a $2.45 \mathrm{GHz}$ wave. The antenna is a rather large structure with its tip at $\mathrm{R}=800 \mathrm{~mm}$ while the radiation pattern has its maximum at $\mathrm{R}=850-860 \mathrm{~mm}$.

We will be mostly concerned with experiments in Argon plasmas in which the neutral density can be as high as $10 \%$ of the electron density. Typically, for WEGA plasmas generated by $2.45 \mathrm{GHz} \mathrm{ECRH}$, there will be two electron components - cold bulk electrons and hot tail electrons. The electron density (plotted in Fig. 1), the bulk electron temperature and the fraction of tail electrons are determined from Langmuir 
probe measurements using a two-temperature model $[3,4]$. Further evidence of fast electrons is seen in the probe current voltage characteristics: the derivative $\mathrm{d} I / \mathrm{d} U$ of the ion saturation current does not vanish even at voltages below $-90 \mathrm{~V}$. The density measurements were confirmed by a central line of sight single line microwave interferometer. The measurements were taken at $\varphi=161^{\circ}$, where the poloidal cross section coincides with the cross section at the antenna position, as well as right in front of the antenna $\left(\varphi=-55^{\circ}\right)$. The antenna influences the plasma density in its vicinity both by heating as well as by partial screening. Since the antenna is mostly an open structure along the magnetic field, it does not act like a limiter. Measurements show that the plasma density at the antenna mouth is below the O-mode cutoff (see Fig. 1) and that the O-mode cutoff and the UHR conversion region lie outside the LCFS. In particular, the typical central WEGA electron density of $\sim 10^{18} \mathrm{~m}^{-3}$ makes the plasma overdense for $2.45 \mathrm{GHz}$ waves since the density cutoff for this frequency is $n_{\text {ecutoff }} \cong 7.5 \times 10^{16} \mathrm{~m}^{-3}$. Therefore only electrostatic EBWs can propagate within this overdense plasma. Since the antenna can only radiate the electromagnetic O-mode and/or X-mode, EBWs can only originate from mode conversion (X-EBW or O-X-EBW) [5]. The direct X-EBW mode conversion is very weak because the incident wave is not only almost linearly polarized along the magnetic field, but also because it is obliquely incident and the density gradient mitigates against the mode conversion.
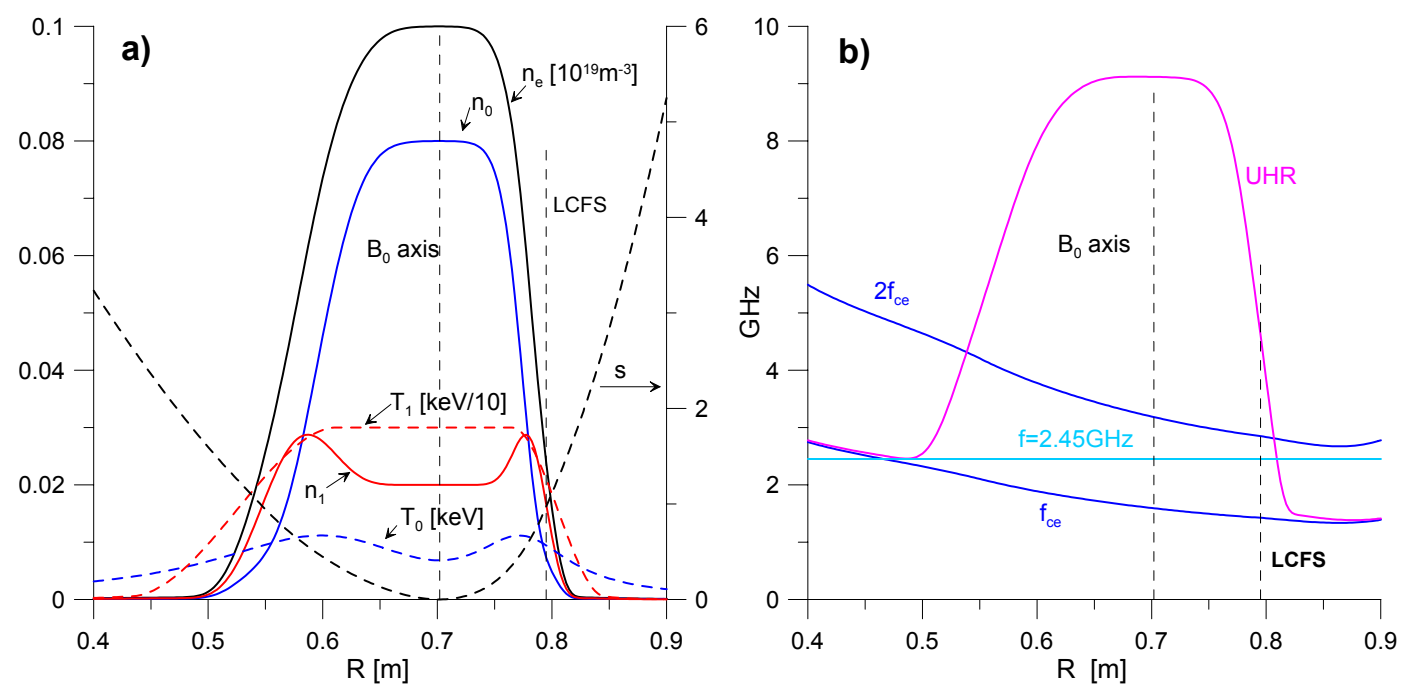

Figure 2. a) The profiles of the density and temperature of the hot and cold electron components of plasma. $s$ is a normalized toroidal flux function corresponding to the equilibrium with $\iota=0.2 ; n_{0}=0.8 \times 10^{-18} \mathrm{~m}^{-3}, n_{1} / n_{e}=0.2, T_{0}=10 \mathrm{eV}$, $T_{1}=300 \mathrm{eV}$. b) Profiles of the characteristic resonances. The parameters are identical to a), $B_{0}=0.65 B_{\text {ce }}$.

In our simulations we use analytic fits to the Langmuir probe data [3], shown in Fig. 2a. Although there is probe data from both the low-field side (LFS) $\left(R>R_{\text {axis }}=\right.$ $0.702 \mathrm{~m})$ and high-field side (HFS) $\left(\varphi=161^{\circ}\right)$, we use only the data from the LFS and then map this onto the magnetic surfaces. 
The analytic fits yield the following fundamental parameter ranges: the central total electron density $0.5 \times 10^{18} \leq n_{0} \leq 4 \times 10^{18} \mathrm{~m}^{-3}$, the bulk electron temperature $5 \leq T_{0} \leq 10 \mathrm{eV}$, the ratio of the hot electron central density to the total electron density $0 \leq R_{\text {tail_bulk }}=n_{1} / n_{\mathrm{e}} \leq 0.2$, and the hot electron temperature $100 \leq T_{1} \leq 300 \mathrm{eV}$. Since the hot electron temperature is difficult to measure, it is assumed to be constant inside the LCFS and to exponentially decay outside the LCFS (see Fig. 2a).
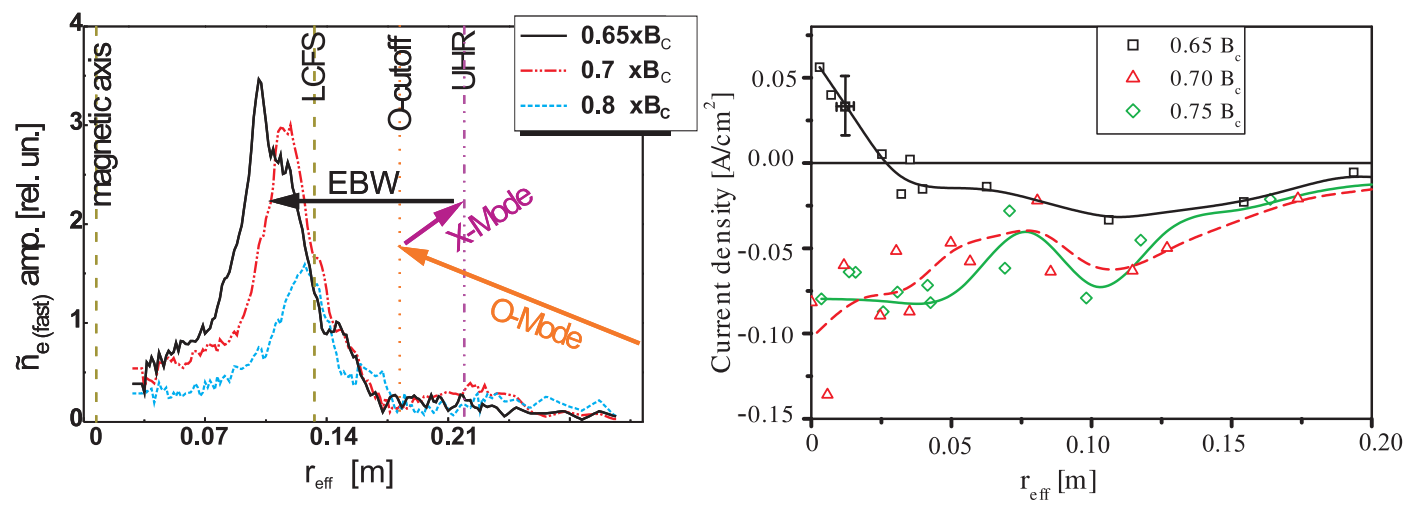

Figure 3. a) Experimental power deposition profiles (heat waves) for different magnetic field strength, as determined from probe measurement of the response to the fast modulated heating power. The modulation in the amplitude of the fast electron density $\tilde{n}_{\text {fast }}$ is used as the signature of power deposition. b) Experimental current density profiles are measured by a movable Rogowski coil for $6 \mathrm{~kW}$ injected modulated power. Figures a) and b) correspond to different series of shots and measurements.

The antenna is located outside the LCFS where the plasma density is below the cutoff density, as shown in Fig. 1. This is consistent with the O-X-EBW conversion mechanism which requires that the electromagnetic waves are incident from an underdense region and propagate towards the UHR and plasma resonance region. We also present in Fig. 2b the radial profiles of the characteristic resonances and cutoffs in the equatorial plane. It is clear that the $2.45 \mathrm{GHz}$ heating frequency is situated between the $1^{\text {st }}$ and the $2^{\text {nd }}$ electron cyclotron harmonics and that for $R \gtrsim 0.82 \mathrm{~m}$ electromagnetic (O- or X-) modes can propagate.

Very strong resonant off-axis power deposition was observed experimentally at the $2^{\text {nd }}$ electron cyclotron harmonic [3]. Using $20 \mathrm{kHz}$ power modulation with coherent detection using differently biased probe arrays, it was observed, that this absorption is reduced and shifted radially outwards by increasing the central magnetic field (Fig. 3a).

It is convenient to characterize the position of a magnetic surface by $r_{\text {eff }}=a_{0} \sqrt{s}$, where $a_{0}$ is the minor radius and $s$ is the magnetic flux label. In the equatorial plane, the transformation between $R\left(R>R_{\text {mag_axis }}\right)$ and $r_{\text {eff }}$ is $R \approx R_{\text {mag_axis }}+$ $r_{\text {eff }}\left(R_{\text {LCFS }}-R_{\text {mag_axis }}\right) / a_{0}$. In particular, for equilibria with $\iota=0.2$ we have $a_{0}=0.1335$ $\mathrm{m}, R_{\text {mag_axis }}=0.70169 \mathrm{~m}, R_{\mathrm{LCFS}}=0.795 \mathrm{~m}$ at the antenna position $\left(\varphi=-55^{\circ}\right)$, where the power deposition and the current density are measured (Fig. 10a).

EBW current drive, which was first predicted by our simulations [6], has now been successfully detected experimentally on WEGA. With $6 \mathrm{~kW}$ of constant power and 6 
$\mathrm{kW}$ of modulated power $(\sim 70 \mathrm{~Hz})$, a counter current $I_{\varphi} \sim-50$ A was measured (i.e., the current direction is opposite to the toroidal magnetic field). This measurement was performed using two independent methods - the short circuited OH-transformer and by Rogowski coils, depicted schematically in Fig. 4. High precision was achieved by making use of the long duration of the WEGA discharge. In a typical $30 \mathrm{~s}$ discharge, $6 \mathrm{~kW}$ magnetron power was modulated $100 \%$ in its amplitude with frequency between $27 \mathrm{~Hz}$ and $90 \mathrm{~Hz}$. A second magnetron sustained the plasma with an additional $6 \mathrm{~kW}$. Since the plasma was stationary for more than $25 \mathrm{~s}$, an average over all modulation cycles gives a precise measurement of the driven current. Both the bootstrap and the Pfirsch-Schlüter currents are negligible in WEGA (very small pressure) and this was confirmed experimentally by observing a linear increase of current with heating power.

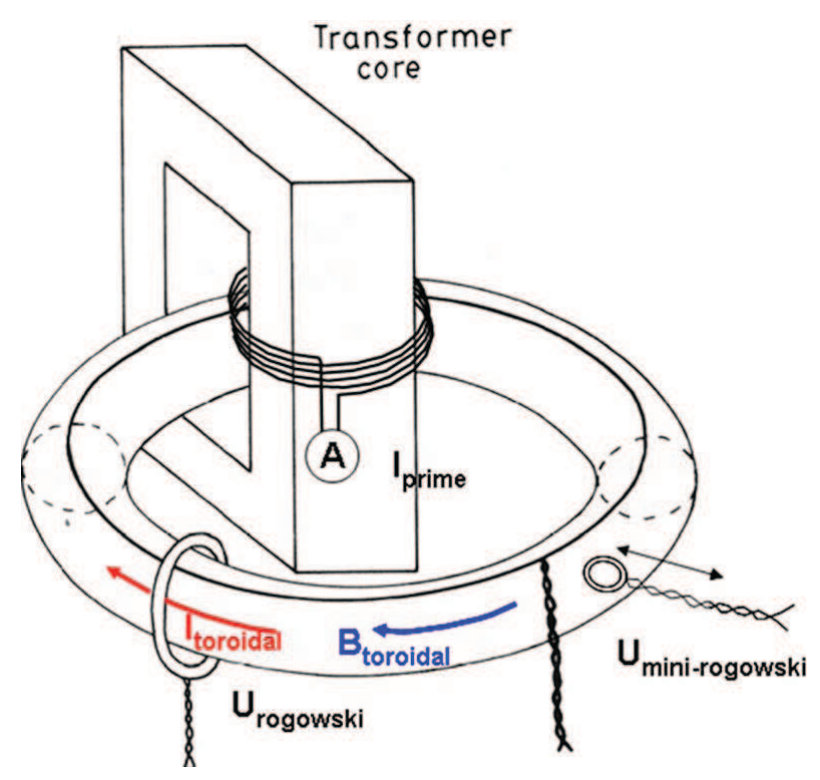

Figure 4. Schematics of the current measurement. The direction of $I_{\text {toroidal }}$ defines the direction of positive current.

Table 1. Toroidal current in WEGA. Temperature of the cold plasma component $T_{0} \approx 10 \mathrm{eV}$

\begin{tabular}{lllll}
\hline Shot number & $B / B_{\text {ce }}$ & Current & Current drive efficiency & Density $\left[10^{17} \mathrm{~m}^{-3}\right]$ \\
\hline 26664 & 0.65 & $-45 \pm 5 \mathrm{~A}$ & $-7.5 \pm 0.83 \mathrm{~A} / \mathrm{kW}$ & $6.4 \pm 1.0$ \\
26665 & 0.7 & $-90 \pm 5 \mathrm{~A}$ & $-15.0 \pm 0.83 \mathrm{~A} / \mathrm{kW}$ & $7.2 \pm 1.0$ \\
26666 & 0.75 & $-60 \pm 5 \mathrm{~A}$ & $-10.0 \pm 0.83 \mathrm{~A} / \mathrm{kW}$ & $5.7 \pm 1.0$ \\
\hline
\end{tabular}

Because the plasma is at a sufficiently low temperature, the current density can be directly measured by current probes, which are moved through the plasma during the discharge. These mini-Rogowski coils consist of a stainless steel tube of $1.3 \mathrm{~mm}$ diameter with a loop of $10 \mathrm{~mm}$ diameter. Inside the tube is a coil with typically 500 windings. Insulating ceramic powder insulates the tube. The results are shown in Fig. 
$3 \mathrm{~b}$, where we see mostly negative and flat current density profile. Current reversal near the magnetic axis is detected for $0.65 B_{\text {ce }}$. This is an unexpected result. Moreover, at this magnetic field we also find the best heating efficiency. It should also be noted that most of the current is driven at the outer radii (although the current density is small here, the plasma volume however is large). Hence, even in this case the total current is negative. The total current measured by the short circuit primary winding of the transformer is shown in Table 1 , together with the simple current drive efficiency $I / P$.

\section{Theory of EBW propagation and damping}

In our model, the electron Bernstein waves that propagate in the two-component WEGA plasma are described by the electrostatic dispersion relation (see e.g. [7], where the electrostatic approximation for EBW's is justified)

$D(\omega, \mathbf{r}, \mathbf{k})=1+\sum_{\alpha=0,1}\left(\frac{2 \omega_{\mathrm{pe}, \alpha}^{2}}{v_{\mathrm{T}_{\alpha}}^{2} k^{2}}\right) \frac{1+\sum_{n} \frac{\omega+i \nu_{\alpha}}{\left|k_{\|}\right| v_{\alpha}} e^{-z_{\mathrm{L}, \alpha}} I_{n}\left(z_{\mathrm{L}, \alpha}\right) Z\left(\tilde{\zeta}_{n, \alpha}\right)}{1+\sum_{n} \frac{i \nu_{\alpha}}{\left|k_{\|}\right| v_{\alpha}} e^{-z_{\mathrm{L}, \alpha}} I_{n}\left(z_{\mathrm{L}, \alpha}\right) Z\left(\tilde{\zeta}_{n, \alpha}\right)}=0$,

where

$$
v_{\mathrm{T}_{\alpha}}=\sqrt{\frac{2 T_{\alpha}}{m_{\mathrm{e}}}}, \quad \tilde{\zeta}_{n, \alpha}=\frac{\omega+n \omega_{\mathrm{ce}}+i \nu_{\alpha}}{\left|k_{\|}\right| v_{\mathrm{T}_{\alpha}}}, \quad z_{\mathrm{L}, \alpha}=\frac{k_{\perp}^{2} v_{\mathrm{T}_{\alpha}}^{2}}{2 \omega_{\mathrm{ce}}^{2}} .
$$

Here the $\alpha$-summation is taken over the hot and cold components of the plasma electrons, collisions are considered in the Bhatnagar-Gross-Krook limit [8] and $Z$ is the plasma dispersion function [9].

WEGA discharges typically consist of singly ionized Argon with a considerable neutral fraction. Therefore, apart from the usual contribution arising from the electronion collisions, the collision frequency must also include the effects of elastic electron-ArI collisions, as well as ionization and excitation of ArI and ArII by electrons. (Here ArI and ArII stand for the neutral Ar atoms and the singly ionized Ar ions, respectively). Thus

$$
\begin{gathered}
\nu_{\alpha}=\nu_{\text {ei }, \alpha}+n_{\mathrm{n}}\left(<v \sigma_{\text {elast }}^{\text {ArI }}>_{\alpha}+\sum<v \sigma_{\text {ioniz }}^{\text {ArI }}>_{\alpha}+\sum<v \sigma_{\text {excit }}^{\text {ArI }}>_{\alpha}\right) \\
+n_{\mathrm{i}}\left(\sum<v \sigma_{\text {ioniz }}^{\text {ArII }}>_{\alpha}+\sum<v \sigma_{\text {excit }}^{\text {ArII }}>_{\alpha}\right)
\end{gathered}
$$

where [8]

$$
\nu_{\mathrm{e}, \alpha}=\frac{4}{3} \sqrt{\frac{2 \pi}{m_{\mathrm{e}}}} \frac{e^{4} Z_{\mathrm{eff}}^{2} n_{\mathrm{e}} \Lambda_{\mathrm{C}}}{T_{\alpha}^{3 / 2}} .
$$

$n_{\mathrm{i}}$ is the ion density, $n_{\mathrm{n}}$ is the Ar neutral density, $Z_{\text {eff }}$ is the effective ion charge number (in our case $Z_{\text {eff }}=1$ ), and $\Lambda_{\mathrm{C}}$ is the Coulomb logarithm. On the right-hand side of Eq. (3), we have summed over the most probable electron collisions, namely elastic collisions with Argon neutrals $\left(\sigma_{\text {elast }}^{\text {ArI }}\right)$, ionization from ArI to ArII $\left(\sigma_{\text {ioniz }}^{\text {ArI }}\right)$ and from ArII to ArIII $\left(\sigma_{\text {ioniz }}^{\text {ArII }}\right)$, as well as excitation of ArI $\left(\sigma_{\text {excit }}^{\text {ArI }}\right)$ and ArII $\left(\sigma_{\text {excit }}^{\text {ArII }}\right)$ but we neglect the excitation or ionization of excited states. As the cross-sections $\sigma_{\text {elast }}^{\text {ArI }}, \sigma_{\text {ioniz }}^{\text {ArI }}, \sigma_{\text {excit }}^{\text {ArI }}, \sigma_{\text {ioniz }}^{\text {ArI }}, \sigma_{\text {excit }}^{\text {ArI }}$ are 
dependent on the energy of the incident electrons (see the IAEA ALADDIN database [10] and M. Hayashi's Ar cross-sections measurements [11]), we must use averaged collision rates $\langle v \sigma\rangle=\int f v \sigma \mathrm{d} v$, where $f$ is the corresponding distribution function. In Fig. 5a we show the temperature profiles of the collision rates. The resulting profiles of the collision frequencies are plotted in Fig. 5b. For the cold plasma component, it is clearly seen from Fig. 5b that the elastic electron-ion collision are dominant: the elastic electron-ion (the dashed curve) $\nu_{\mathrm{ei}, 0} / \omega$ does not differ substantially from $\nu_{0} / \omega$. However, for the hot component, the electron-ion elastic collisions are very weak $\left(\nu_{\mathrm{e} i, 1} / \omega \sim 10^{-6}\right)$ and $\nu_{1} / \omega$ is dominated by the process of excitation of argon ions by electrons. Taking into account that $\omega_{\mathrm{pe}, 1}^{2} / \omega_{\mathrm{pe}, 0}^{2} \leqslant 0.2$, the contribution to the imaginary part of the dispersion relation (1) from $\nu_{1}$ is small because $v_{0} \gg \nu_{1}\left(T_{0} / T_{1}\right)\left(\omega_{\mathrm{pe}, 1}^{2} / \omega_{\mathrm{pe}, 0}^{2}\right)$. Thus the collisional wave damping is determined by the elastic electron-ion collisions in the cold component. On the other hand, in the hot component contribution to the current (Eq. (9)), inelastic collisions of electrons with argon ions are important. In the simulations, we use the full collisional frequencies (3) and do not make any truncations.

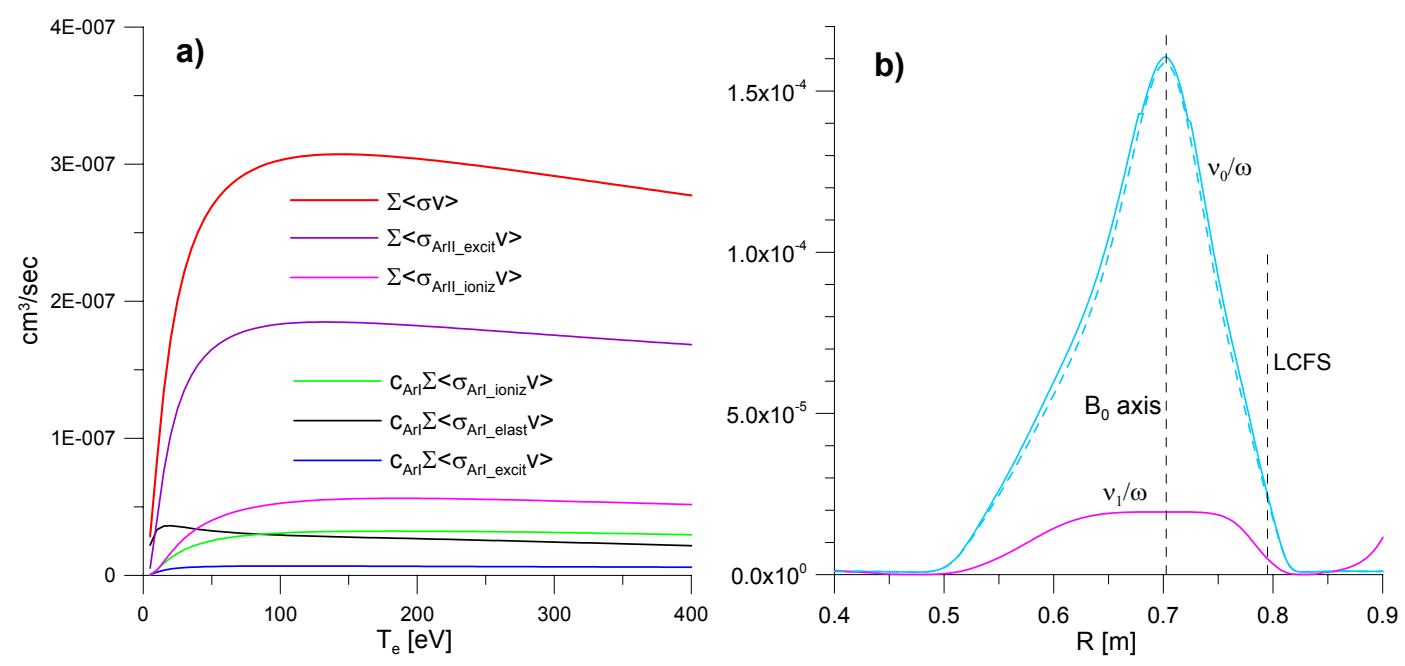

Figure 5. a) Profiles of averaged collision rates for the electron-ArI/ArII collisions, $n_{\mathrm{n}}=0.1 n_{0}$. b) Profiles of normalized collision frequencies $\nu_{0} / \omega$ and $\nu_{1} / \omega$ for the cold and the hot electron components. For profiles of $n_{\alpha}, T_{\alpha}$ see Fig. 2a.

The propagation of the short wavelength electron Bernstein waves in a weakly inhomogeneous plasma can be studied in the framework of the WKB theory, with the ray trajectory governed by the Hamiltonian equations [12]:

$$
\begin{aligned}
& \frac{\mathrm{d} \mathbf{r}}{\mathrm{d} t}=-\frac{\partial \bar{D}}{\partial \mathbf{k}} / \frac{\partial \bar{D}}{\partial \omega}=\frac{\partial \omega}{\partial \mathbf{k}}=\mathbf{v}_{g}, \\
& \frac{\mathrm{d} \mathbf{k}}{\mathrm{d} t}=\frac{\partial \bar{D}}{\partial \mathbf{r}} / \frac{\partial \bar{D}}{\partial \omega} .
\end{aligned}
$$

Here $\bar{D}$ stands for $\Re(D(\omega, \mathbf{r}, \mathbf{k}))$ where, in $D$, we neglect the collisional terms, i.e.

$$
\bar{D}(\omega, \mathbf{r}, \mathbf{k})=1+\sum_{\alpha=0,1} \frac{2 \omega_{\mathrm{pe}, \alpha}^{2}}{v_{\mathrm{T}_{\alpha}}^{2} k^{2}}\left(1+\sum_{n} \frac{\omega}{\left|k_{\|}\right| v_{\mathrm{T}_{\alpha}}} e^{-z_{\mathrm{L}, \alpha}} I_{n}\left(z_{\mathrm{L}, \alpha}\right) \Re\left(Z\left(\zeta_{n, \alpha}\right)\right)\right),
$$


where $\zeta_{n, \alpha}=\left(\omega+n \omega_{\text {ce }}\right) /\left|k_{\|}\right| v_{\mathrm{T}_{\alpha}}$. Contributions to $\Re(D)$ from the collisions are very small, being proportional to $\nu^{2} / \omega^{2}$.

These equations describe the motion of the wave packet along the ray. The individual amplitude of the rays are determined from the antenna radiation pattern from each lobe and these amplitudes are evolved independently. Following [13], we neglect the effect of ray focusing and consider the power deposition into the plasma as the decay of the incident power along the ray as it propagates in time

$$
\frac{\mathrm{d} P}{\mathrm{~d} t}=-2 \gamma P
$$

where $\gamma$ is the time damping decrement (the imaginary part of $\omega$ ) given by

$$
\gamma=-\Im(D /|\partial \bar{D} / \partial \omega|) \text {. }
$$

The power deposition is determined by integrating (7) along the ray trajectory $(5 a)$.

The wave damping is accompanied by a toroidal current due to the creation of an asymmetric plasma resistivity [14]. To determine the generated current density we employ a model proposed by F.R. Hansen et al. [15], but here generalized to a two component plasma with multiple electron cyclotron resonances:

$$
J=-\sum_{\alpha=0}^{1} \sum_{n=0}^{\infty} \frac{e}{m_{\mathrm{e}} v_{\mathrm{T}_{\alpha}} \nu_{\alpha}} \frac{3}{2}\left(\frac{v_{\mathrm{res}, n}}{v_{T_{\alpha}}}\right)^{2} \frac{\left|v_{\mathrm{res}, n}\right|}{v_{\mathrm{res}, n}} \frac{4}{5+Z} P_{\mathrm{d}, \alpha, n} .
$$

Here, the resonant velocity for the $\mathrm{n}^{\text {th }}$ harmonic is $v_{\text {res }, n}=\left(\omega-n \omega_{\text {ce }}\right) / k_{\|}$. To determine that part of the power density deposited to the resonant particles $P_{\mathrm{d}, \alpha, n}$ we must determine the partial non-collisional damping rates

$$
\gamma_{\alpha, n}=\left(\frac{2 \omega_{\mathrm{pe}, \alpha}^{2}}{v_{\mathrm{T}_{\alpha}}^{2}\left(k_{\perp}^{2}+k_{\|}^{2}\right)}\right) \frac{\omega}{\left|k_{\|}\right| v_{T_{\alpha}}} e^{-z_{\mathrm{L}, \alpha}} I_{n}\left(z_{\mathrm{L}, \alpha}\right) \Im\left(Z\left(\zeta_{n, \alpha}\right)\right)
$$

and integrate the evolution equation for each ray,

$$
\frac{\mathrm{d} P_{\alpha, n}}{\mathrm{~d} t}=-2 \gamma_{\alpha, n} P .
$$

Note that the effect of collisions is not included in the expression for $\gamma_{\alpha, n}$, as they do not contribute to the current, whereas the term $P$ in the right-hand side of (11) is influenced by the collisions since it is the solution of (7).

The deposited power density profile is given by

$$
P_{\mathrm{d}, \alpha, n}\left(r_{\mathrm{eff}}\right)=\sum_{\text {rays }} \frac{\Delta P_{\alpha, n}\left(r_{\mathrm{eff}}\right)}{\Delta V\left(r_{\mathrm{eff}}\right)} C_{\mathrm{O}-\mathrm{X}-\mathrm{EBW}} P_{\mathrm{inc}},
$$

where $\Delta V\left(r_{\text {eff }}\right)$ is the volume enclosed by two adjacent magnetic surfaces $s\left(r_{\text {eff }} \pm \Delta r_{\text {eff }} / 2\right)$ and $\Delta P_{\alpha, n}\left(r_{\text {eff }}\right)$ is the power deposited within this volume. $C_{\mathrm{O}-\mathrm{X}-\mathrm{EBW}}$ is the O-X-EBW conversion efficiency and $P_{\text {inc }}$ is the incident power associated with the ray. 


\section{Simulation results}

Our solution procedure is the following: We first consider the antenna part of the problem. The radiation pattern from each lobe is discretized by a bundle of 41 points. Using straight line propagation of these points onto the plasma surface, we obtain the "dots" in Fig. 6. Now we consider the computation of O-X-EBW mode conversion efficiency. This is performed for each "dot" using a 1D full wave solution of the electromagnetic wave equation in a plane stratified slab constructed in the vicinity of the "dot". Finally, having determined the mode conversion efficiency at each "dot", we perform 3D electrostatic ray-tracing computations from the UHR parameters near that particular "dot" to determine the power deposition and current drive efficiency along the ray trajectory. Finally, the results from each individual ray are summed, according to Eq. (12), yielding the result for the whole beam.

\subsection{Efficiency of the $O-X-E B W$ conversion}

The O-X-EBW mode conversion in WEGA was demonstrated experimentally by probe measurements of both the amplitude and phase of the wave field in the conversion region and supported by two dimensional full-wave calculations [3]. However 2D simulations are computationally quite expensive. Because the mode conversion region is thin it is sufficient to use a 1D slab model of the O-X-EBW conversion efficiency. This has also been our approach for determining the conversion efficiency for EBW emission in spherical tokamaks $[16,17]$. For each ray we determine the spot on the plasma surface. Typically, we would take the plasma surface to be the LCFS. But for $2.45 \mathrm{GHz}$ waves the LCFS is too deep within the plasma, lying past the upper hybrid resonance and plasma cut-off. Hence, for computational reasons only, we take the plasma surface to be an open surface with $s=2.5$.

At the spot (or "dot") we construct an auxiliary plane stratified plasma slab, which is inhomogeneous along $\nabla s$. The full wave equation for the propagation of an electromagnetic wave into a cold plasma is solved in this slab geometry, with ad hoc collisions introduced to smooth out the cold dispersion singularities (in our simulations, we choose $\nu / \omega=10^{-4}$ ). Both O-X-EBW (i.e., O-mode - slow X-mode - electron Bernstein wave) and X-EBW (fast X-mode - slow X-mode - EBW) conversions are automatically included in the full wave solution. Also the possibility of slow X-mode fast X-mode tunneling is automatically taken into account. Our cold plasma code has been benchmarked against a warm plasma code [18] in which finite Larmor radius effects are taken into account. We find that the cold plasma code deviates only from the warm plasma code when the plasma profiles are non-monotone and thus introduce multiple UHR's. The full wave equations are solved by an adaptive finite element method [17]. The incident wave is typically a mixture of both $\mathrm{O}$ - and X-modes. The efficiency of the conversion of the incident wave to EBW is determined from the ad-hoc collisional absorption of the waves at the UHR region (for details see [17]). For the WEGA plasmaantenna system we find a conversion efficiency of $20-30 \%$ for a first pass. The reflected 


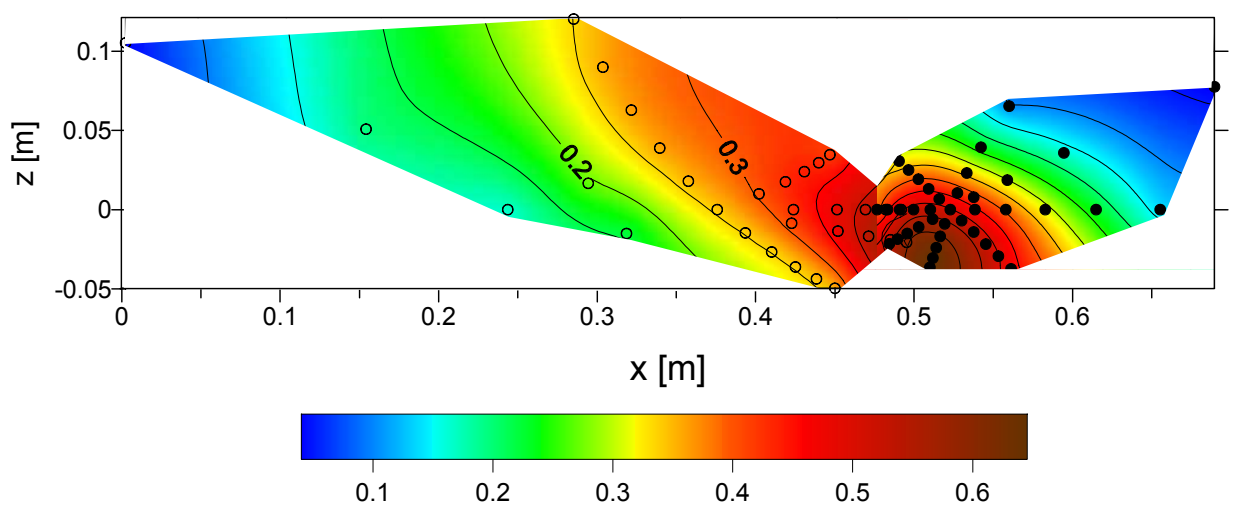

Figure 6. O-X-EBW conversion efficiency for the WEGA $2.45 \mathrm{GHz}$ antenna. For plasma and equilibrium parameters see Fig. $2 \mathrm{a}$ and $B_{0}=0.65 B_{\text {ce. The coordinate }}$ system is the same as in Fig. 7a. In the simulations, the radiation lobes of the antenna are represented by bundles of straight rays. The black dots represent the spots of the intersection of these rays with the plasma surface $(s=2.5)$ from the right antenna lobe while the corresponding open circles are the intersection spots coming from the left lobe rays. Some of these left lobe rays do not even intersect the plasma. The color scale displays the mode conversion efficiency (normalized to 1). In the white area, the intensity of the incident wave is very small and the mode conversion efficiency here is set to zero.

power can be experimentally reduced to below $5 \%$ by introducing a feed back stub tuner, but the power losses from the absorption inside the ports and from the resistivity of the wall are unknown and difficult to estimate. A total plasma radiation of up to $50 \%$ of the input power has been measured by a 12 channel bolometer. Therefore the assumption of a $30 \%$ conversion efficiency in the first pass is quite reasonable. Further absorption will occur because of multiple reflections between the plasma and the vacuum vessel but this absorption will most probably just heat the plasma periphery and not contribute to the current drive.

Now the mode conversion efficiency for linearly polarized waves is typically below $60 \%$, but for the WEGA stellarator our computed conversion efficiency is only $20-30$ $\%$. This reduction in the mode conversion efficiency can be attributed to the following factors:

(i) The left beam is incident nearly parallel to the magnetic field.

(ii) The angle between the E-field and the equatorial plane is $0^{\circ}$. This is not the optimum orientation because of the slope in the density gradient. With the WEGA stellarator density gradient, the optimum polarization is $15^{\circ}$. Thus, with $0^{\circ}$ orientation and with the linear polarization (the oblique O-mode is practically circularly polarized), there occur massive reflections. It is expected that these waves will bounce between the plasma and the vessel wall several times before being absorbed. However, in our simulations, we only consider the first pass of the waves as they emerge from the antenna.

(iii) Some rays from the left beam of the WEGA antenna do not even intersect the 
plasma.

On the other hand, since the wavelength is comparable to the dimensions of the plasma itself, the O-mode can be transmitted through the plasma cutoff for a broad range of angles of incidence.

In Fig. 6, we show the projection of the mode conversion efficiency contours onto the plasma surface (see also Fig. 7a). One sees that the right lobe of the radiation pattern is converted with around $50 \%$ efficiency (the angle between the normalized wave vector $\vec{N}=\vec{k} / k_{\text {vac }}$ of the central ray of the right lobe and the magnetic field $\vec{B}$ at the plasma surface is $36^{\circ}$ ). However, for the left lobe, the conversion efficiency is only $10-20 \%$ (the angle between $\vec{N}$ and $\vec{B}$ is now $13^{\circ}$ ).

\subsection{Ray tracing}
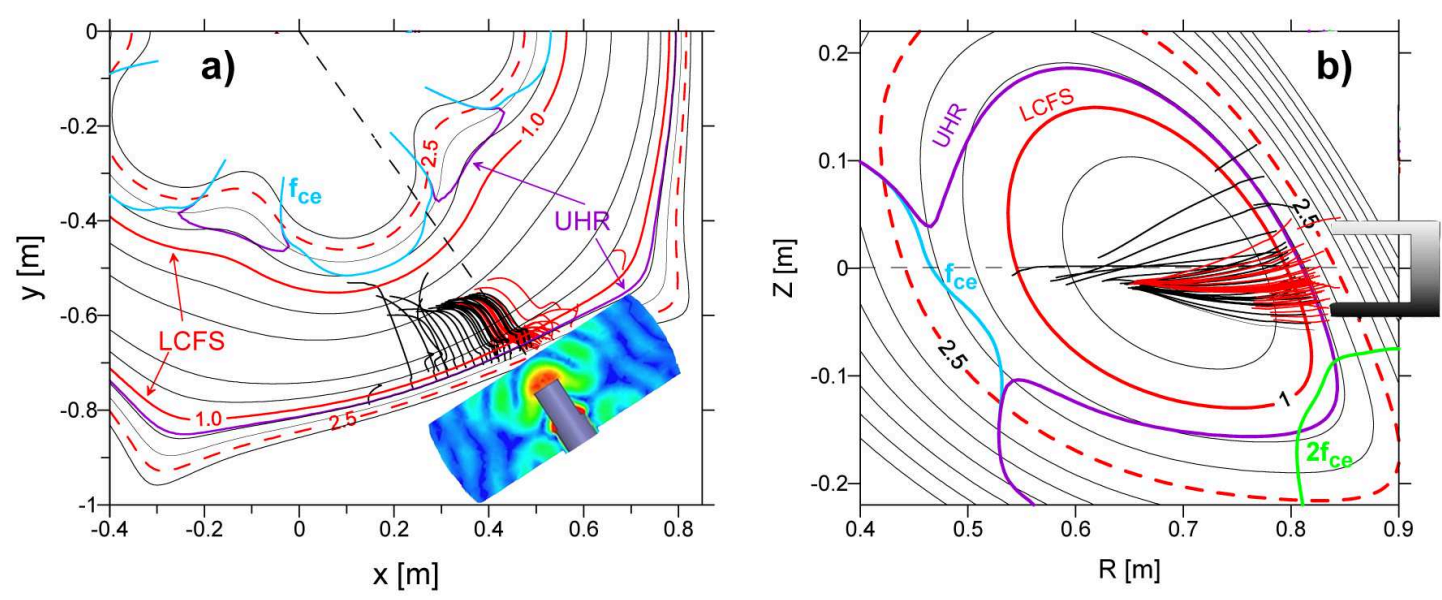

Figure 7. a) The equatorial cross-section of the WEGA plasma with the position of the antenna and the EBW rays (the black rays are from the left lobe while the red rays are from the right lobe). The contours of UHR, $f_{\text {ce }}$ and $2 f_{\text {ce }}$ (purple, blue, green) correspond to a $2.45 \mathrm{GHz}$ wave while the black and red contours are of the magnetic surfaces $s$. The full wave antenna pattern is only illustrative and, in the simulation, it serves to determines the intensity of the irradiation of the plasma surface $(s=2.5$ is used as an auxiliary surface of the plasma in the simulations). b) The poloidal crosssection of the WEGA plasma with EBW rays and a side view of the antenna. The plasma parameters and equilibrium are shown in Fig. 2 a. $B_{0}=0.65 B_{\text {ce. }}$.

The incident beam from the antenna is modeled by 41 rays. The ray propagation from the antenna to the plasma surface is taken to be a straight line and its intersection with the plasma surface is denoted by a "spot" (see Fig. 6). To initialize the ray for ray tracing within the plasma, we first determine the root of the EBW dispersion relation (6) in the vicinity of the UHR, adjacent to the spot on the plasma surface. $N_{\|}$is given here by the projection of the incident wave vector to the direction of the magnetic field at UHR. The power associated with each ray is initialized by the corresponding value of the experimentally measured antenna radiation pattern at that spot location weighted 
by the corresponding O-X-EBW conversion efficiency. In Fig. 7a we show the equatorial cross-section of WEGA with the position of the antenna. As the rays are incident on the plasma surface under dissimilar angles, they will eventually be all focused in the same direction (i.e., along the magnetic field).

From the ray trajectories in the poloidal cross-section (Fig. 7b) we can deduce two very important consequences for the ray properties and the final power deposition. First, the $2^{\text {nd }}$ electron cyclotron harmonic is close to the ray trajectory at the plasma periphery so waves can be damped here provided a warm electron component is present. Second, the $1^{\text {st }}$ electron cyclotron harmonic is concave so that the rays will oscillate about the midplane as will $N_{\|}$, the component of the normalized wave vector parallel to the magnetic field [19]. This will have a major impact on current generation in the plasma.

\subsection{Development of $N_{\|}$}

Since $N_{\|}$plays a crucial role in the resonant damping of the waves, the development of $N_{\|}$along the rays in the WEGA plasma for both lobes of the radiation pattern is shown in Fig. 8. The hot electron component has an important effect on the development of $N_{\|}$along the ray (see Fig. 8a, Fig. 9b) $-\left|N_{\|}\right|$reaches large values $\left(N_{\|} \sim-4\right)$ near the plasma periphery and it is this that makes possible the $2^{\text {nd }}$ harmonic absorption.
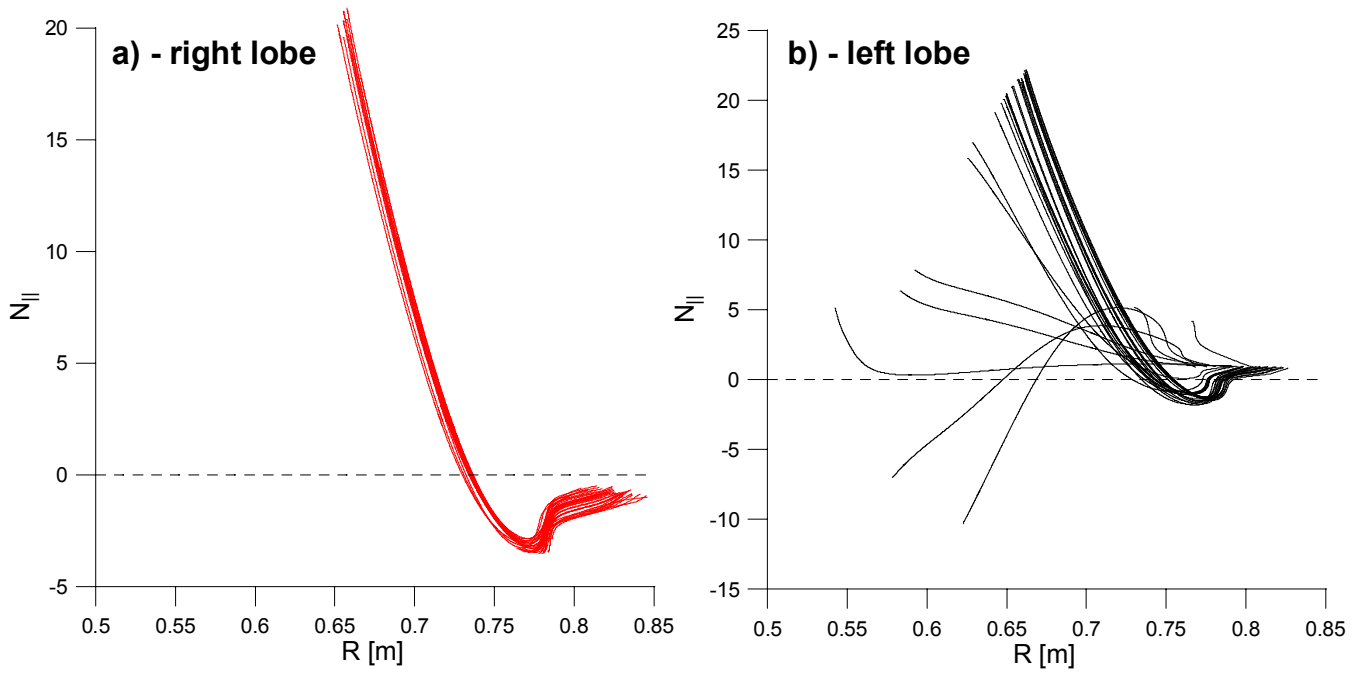

Figure 8. a) The development of $N_{\|}$along the EBW ray trajectories from the right lobe. The plasma parameters are as in Fig. 2a, using the standard hot electron density profile $n_{1}$. For the modified $n_{1}$ profiles, all EBW rays are absorbed at the plasma boundary and the trajectories end at $R=0.77 \mathrm{~m}$. b) The development of $N_{\|}$ along the EBW rays from the left lobe using the standard hot electron density profile $n_{1}$. For the modified $n_{1}$ profiles some EBW rays are absorbed at the plasma boundary and end at $R=0.77 \mathrm{~m}$.

To explain the synergy between the left and right antenna lobe rays at the plasma centre we present in Fig. 9a a comparison of the $R Z$ ray trajectories and the development 
of the corresponding $N_{\|}$along that ray-see also Fig. 8. The central rays from both the left and the right lobe initially start below the equatorial plane and move further below it. As a consequence, $N_{\|}$starts to decrease irrespective of whether $N_{\|}>0$ or $N_{\|}<0$. When the ray trajectory swings back to the equatorial plane $N_{\|}$starts to grow. Both rays have large positive $N_{\|}$in the plasma centre and are damped before they reach the equatorial plane. Thus, while the rays tend to oscillate they only go through a half cycle before they are damped. The large value of $N_{\|}$is caused by the extremely high magnitude of $N_{\perp}$ in a very cold plasma because for EBW's $k_{\perp} \rho_{\mathrm{L}} \approx 1$ ( $\rho_{\mathrm{L}}$ is the electron Larmor radius) and also from the projection of the resulting wave vector on the strongly curved magnetic field. Some rays from the left lobe start above the equatorial plane and end up with $N_{\|}<0$. Therefore, to avoid current generation, it is sufficient to have two antennas, one aimed below the equatorial plane and the other above it.

\subsection{Two component plasma}

We study the effect of the hot electron component on the $2.45 \mathrm{GHz}$ EBW as regards heating and current drive in WEGA for a central magnetic field $B_{0}=0.65 B_{\text {ce }}$. The experimentally observed off-axis power deposition (Fig. 3a) and current reversal (Fig. $3 \mathrm{~b}$ ) can be only explained by the presence of a hot electron component in the WEGA plasma. These hot electrons are generated by the $2.45 \mathrm{GHz}$ ECRH and have densities approaching as high as $20 \%$ of plasma electron density.
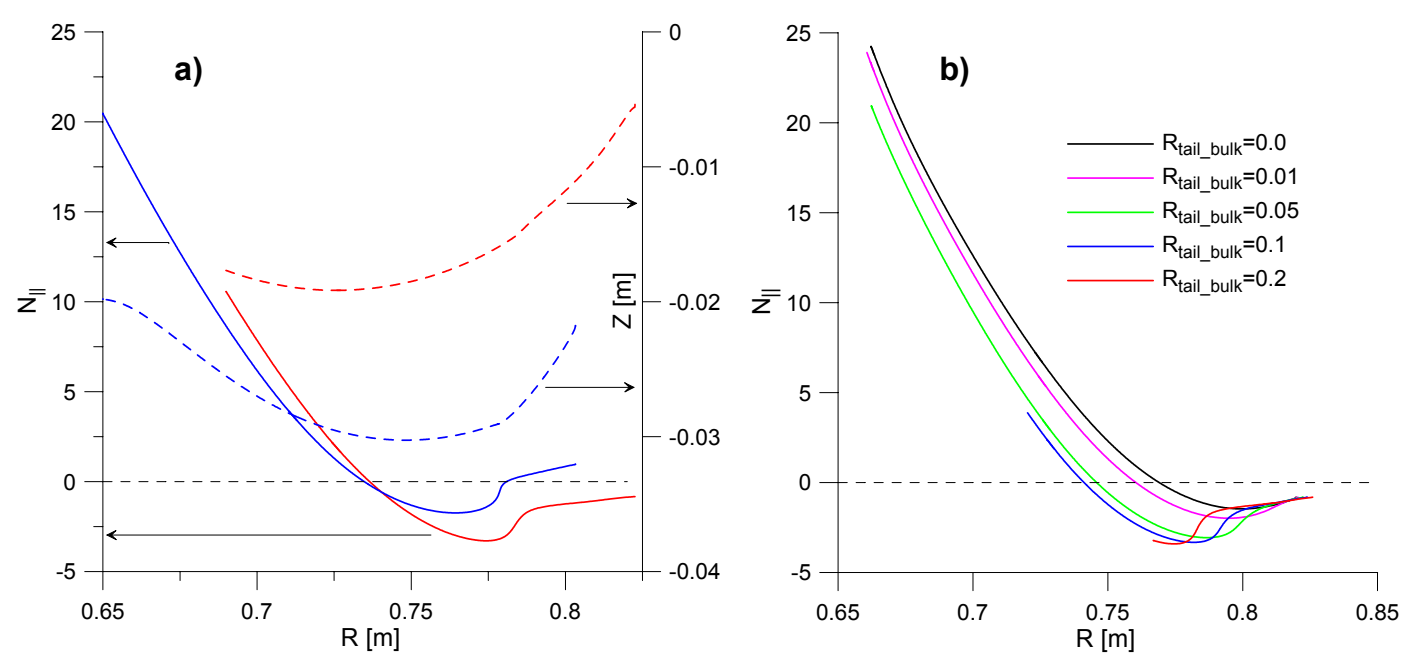

Figure 9. a) The evolution of the central EBW ray from the left lobe (blue) and the right lobe (red). Solid Curves : $N_{\|}$. Dashed curves: the $R Z$ ray trajectory. b) Profiles of $N_{\|}$along the central EBW rays from the right lobe corresponding to a different population of the hot electron component (as in Fig. 10a.)

In a cold one component plasma, the power is deposited at the plasma centre on the first electron cyclotron harmonic. A negative current is generated at this location, see Fig. 10b. However, even a small hot component will cause an off-axis power deposition since the absorption will now occur on the second harmonic at the plasma periphery, 
see Fig. 10a. The generation of the negative current is also shifted off-axis. As the concentration of the hot electron component increases, the off-axis absorption becomes more effective and shifts slightly inward. Consequently, the negative current in the centre diminishes. This behaviour is caused by two effects of the hot electron component. First, the wave damping is more efficient at higher temperatures. Second, the ray evolution is significantly affected by the presence of the hot electrons.
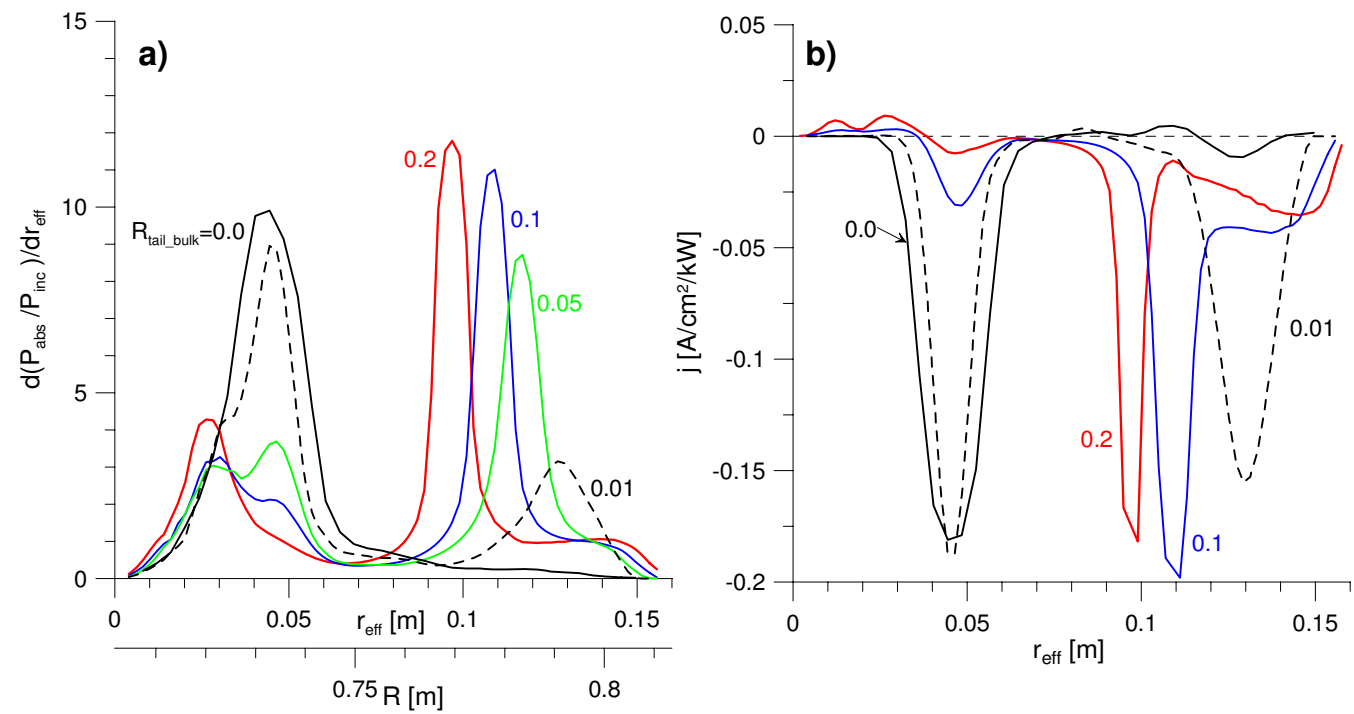

Figure 10. a) Profiles of the deposited power for different populations of the hot electron component $\left(R_{\text {tail_bulk }}=n_{1} / n_{\mathrm{e}}\right) . B_{0}=0.65 B_{\text {ce }}, T_{0}=10 \mathrm{eV}, \mathrm{T}_{1}=300 \mathrm{eV}$, $\mathrm{n}_{\mathrm{e}}=10^{18} \mathrm{~cm}^{-3}$. The $R$-scale corresponds to the antenna position $\left(\varphi=-55^{\circ}, Z=0\right)$. b) The corresponding profiles of the current density for the corresponding hot electron population as in a).

The dependence of the power absorption on the density of the hot electron component serves also as an explanation for the build up of the hot component itself. A small number of hot electrons on the periphery initiate the absorption, which, as the density of the hot electrons increases, is shifted inward (see Fig. 10a). This process reduces the hot particle losses and thus steady state can be reached.

The current reversal at the plasma centre, as will be described later, is also caused by damping on the second harmonic. This current reversal requires a sufficiently high hot electron density. (see Fig. 10b).

\subsection{Power deposition and current density profiles in a $B_{0}$-parameter scan}

Experiments on WEGA indicate that the profiles of the deposited power and that of the current density both depend strongly on the magnitude of the magnetic field at the magnetic axis $B_{0}$ (see Fig. 3a). A comparison of the effects of a $B_{0}$-parameter scan on the profiles obtained by simulations and those from experiment can benchmark our simulation code and thus permit an explanation of the experimental results. 

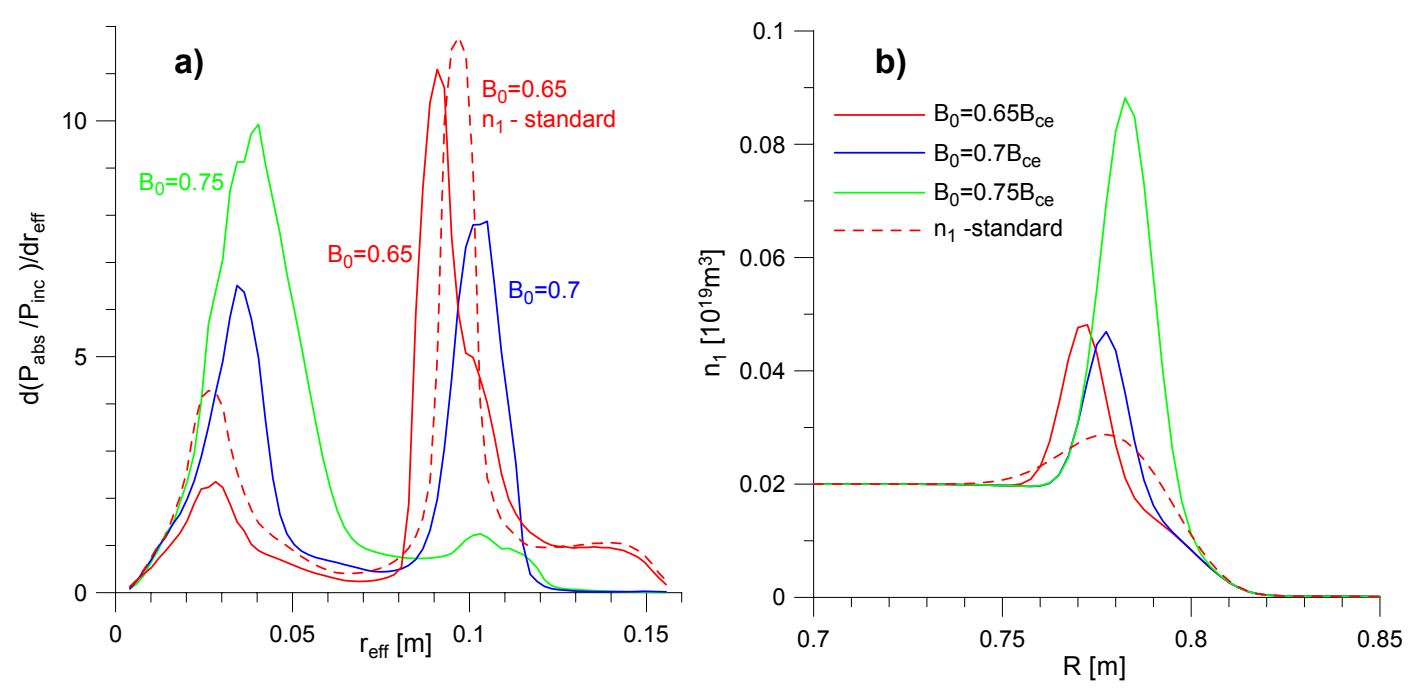

Figure 11. a) The effect of central magnetic field strength $B_{0}$ on the power deposited in the WEGA plasma. The plasma parameters and equilibrium are given in Fig. 2a, with $Z_{\text {eff }}=1$, and $10 \%$ Ar neutrals. b) The hot electron component density profiles used in the simulation of power deposition at different magnetic fields. The standard profile is averaged and used as an initial estimate in the iterative process.

In the WEGA experiment, the off-axis heating on the $2^{\text {nd }}$ harmonic was prominent and the deposited power was clearly related to the existence of hot electrons. To achieve better agreement between simulation and experiment, the hot electron $n_{1}$ profiles were iteratively modified. Since the fast electrons are generated by the ECRH itself, the $n_{1}$ peak must coincide with the power deposition peak for a particular $B_{0}$. The resulting fast electron density profiles are shown in Fig. 11b. The modified $n_{1}$ profiles yield much better agreements between simulated and experimental power deposition in the case of $B_{0}=0.65 B_{\text {ce }}$ and $B_{0}=0.7 B_{\text {ce }}$, see Fig. 11a. However, even a sharply peaked $n_{1}$ profile did not yield substantial off-axis power deposition for the case of $B_{0}=0.75 B_{\text {ce }}$. The simulations also show a significant central deposition caused by the absorption on the $1^{\text {st }}$ harmonic. However, the central deposition was not investigated experimentally.

In the experiment, one typically measures a negative current (i.e., counter to the toroidal magnetic field). Very interestingly, a current reversal at the plasma centre was observed for $B_{0}=0.65 B_{\text {ce }}$. Our simulations recovered the same current reversal behaviour at the plasma centre for this field, Fig. 13a. The peaked character of the current profiles (Fig. 10b) results from the peaked power deposition (i.e., the generated current is localized to those positions at which the power is absorbed). The experimental profiles are smoother than those from our simulations-however, this smoothing is probably caused by the finite size of the probe and time averaging of the data. In our simulations, we neglect the effects of plasma turbulence or current diffusion. Both of these effects would typically smooth out the (averaged) profiles. Our simulations predict a total negative current with values between $-15 \mathrm{~A} / \mathrm{kW}$ to $-5 \mathrm{~A} / \mathrm{kW}$. This is in agreement with experiments. 

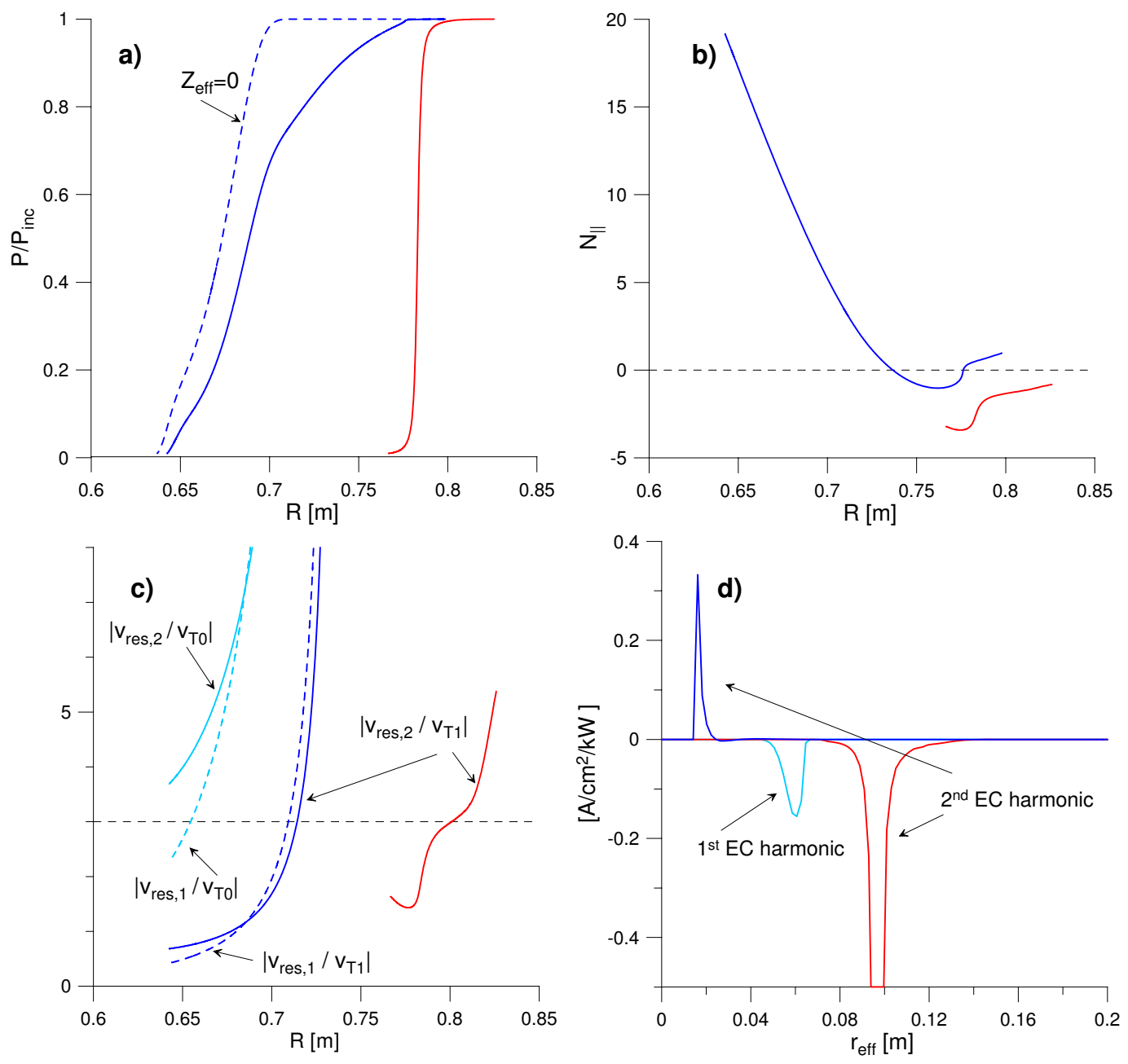

Figure 12. a), b) and c) Radial profiles of several parameters along the central EBW rays from the right lobe (red) and the left lobe (blue). $B_{0}=0.65 B_{\mathrm{ce}}$ and the remaining parameters as in Fig. 2a. a) Incident powers, dashed line - left lobe with collisions ignored. b) $N_{\|}$. c) Normalized resonant velocities (see eq. (9)). The missing three velocities for the right lobe are off scale. The full lines correspond to the $2^{\text {nd }}$ harmonic, while the dashed lines to the $1^{\text {st }}$, blue lines - hot component, light blue cold component. d) The radial profiles of the partial current density corresponding to the individual electron plasma components along the central EBW rays.

\subsection{Explanation of the current reversal}

As is seen from Fig. 13a, the current density shows current reversal at the plasma centre for the lowest magnetic field $B_{0}=0.65 B_{\text {ce }}$. To understand this behaviour, we first consider the damping of the central rays from the right and left lobes of the antenna. In Fig. 12, we show, for both lobes, the radial profiles of the normalized power $P / P_{\text {inc }}$, the parallel component of the refractive index $N_{\|}$and the ratios $\left|v_{\mathrm{res}, n} / v_{\mathrm{T}_{\alpha}}\right|$ of the resonant velocity ( $n=1,2$ respectively), for the cold and the hot electron plasma components $\left(\alpha=0,1\right.$ respectively). Note, that the absorption is significant only if $\left|v_{\text {res }, n} / v_{\mathrm{T}_{\alpha}}\right|<3$. While collisional damping does not contribute to the current drive, it must be considered 
because it influences the evolution of the power along the ray trajectory. In Fig. 12a, we see that collisions are important only at the plasma centre and influence the central ray of the left lobeł. The central ray of the right lobe (whose incident direction is antiparallel to the magnetic field at the plasma boundary, i.e., with $N_{\|}<0$ ) is absorbed by the hot electrons at the $2^{\text {nd }}$ harmonic on the low field side (LFS) plasma boundary. Nearly all the rays from the right lobe (see Fig. 8a) are similarly absorbed at the plasma boundary, and because $N_{\|}<0$ in this region, the current at the periphery is negative (red line in Fig. 12d). The rays from the left lobe (see Fig. 8b) are incident in the direction of the magnetic field. Thus, these rays start with $N_{\|}>0$ and even if $N_{\|}$ decreases it does not reach the large negative values needed for appreciable damping to occur at the plasma boundary. Hence, these rays are only slightly collisionally damped in this region and they penetrate to the plasma centre. Here, $N_{\|}$is positive and very large so ray damping by the hot electrons is possible on both $1^{\text {st }}$ and the $2^{\text {nd }}$ harmonics. From Fig. 12c, one sees that the $2^{\text {nd }}$ harmonic damping dominates and a positive current is generated (blue line in Fig. 12d). As these rays penetrate into the HFS of the plasma, some power is absorbed on the $1^{\text {st }}$ harmonic, and so will generate an off-axis negative current (light blue line in Fig. 12d).
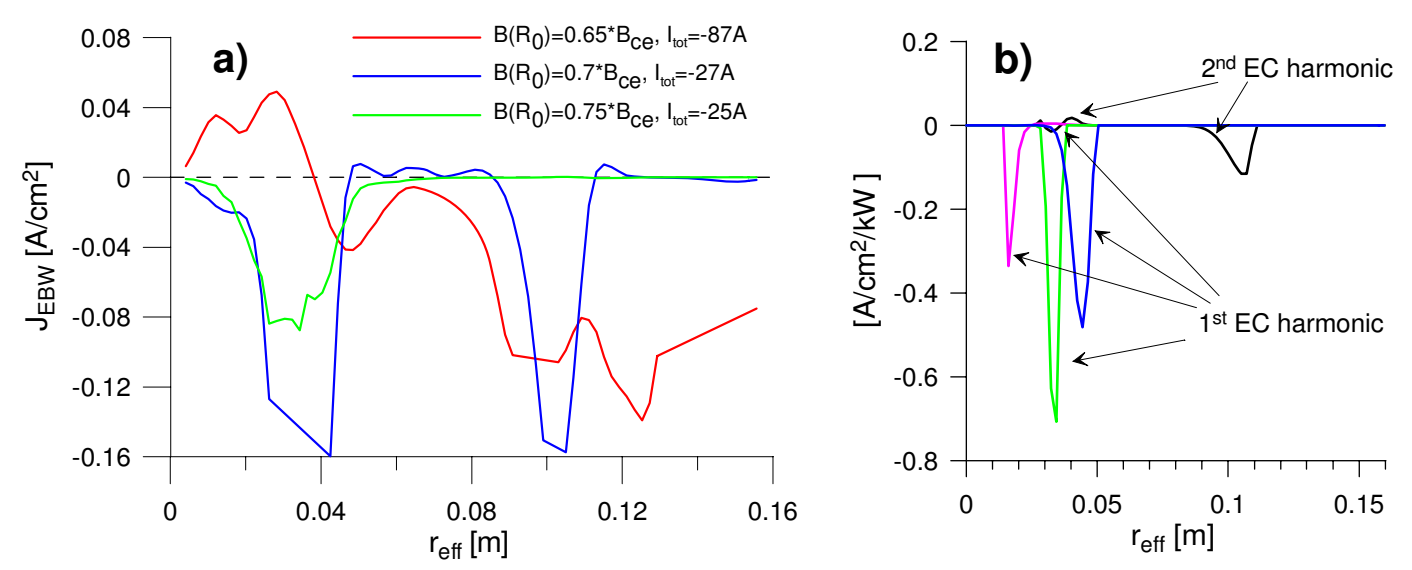

Figure 13. a) The current density density profiles as a function of the central magnetic field $B_{0}$. The incident power is $6 \mathrm{~kW}$ with the standard hot electron density profile $n_{1}$. The remaining parameters are given in Fig. 10a. b) The radial profiles of the partial current density corresponding to the individual electron plasma components along the central EBW rays. The right lobe waves are absorbed by the hot electron component and are shown in black, while those waves absorbed by the cold electron component are in green. The left lobe waves absorbed by the hot electron component are shown in purple, while those by the cold electron component in blue. $P_{\text {inc }}=1 \mathrm{~kW}$, $C_{\mathrm{O}-\mathrm{X}-\mathrm{EBW}}=1, B_{0}=0.7 B_{\mathrm{ce}}$.

$\ddagger$ In this aspect the WEGA plasma differs substantially from the situation in NSTX and other devices having the UHR region displaced outside the last closed flux surface. These devices suffer from very poor coupling as a result of the collisional damping. The WEGA edge - or, more precisely, the UHR layer - is cold but also dilute, resulting in a local collisional frequency $\nu_{\mathrm{e}, \mathrm{i}} \ll \omega$, and thus in negligible collisional losses (see also Fig. 3a). Compared to WEGA, the NSTX edge is characterized by a higher $\omega$ and a substantially denser plasma resulting in higher $\nu_{\mathrm{e}, \mathrm{i}} / \omega$. 
For higher magnetic fields, the damping on the $2^{\text {nd }}$ harmonic is weaker (since the $2^{\text {nd }}$ harmonic retreats further away from the plasma on the LFS). Even if some rays induce positive current the massive absorption on the $1^{\text {st }}$ harmonic at the plasma centre on both the cold and the hot components produces a negative current density everywhere (see Fig. 13b). Therefore, the current reversal is present only for $B_{0}=0.65 B_{\text {ce }}$, in agreement with the experiment, and is not seen for higher magnetic fields.

\section{Conclusions}

We conclude that EBW propagation, power absorption and current drive, in the WEGA stellarator discharges under consideration here, are well described by our simulations using the AMR code. Our simulations reproduce the off axis power deposition seen in the experiment as well as the outward shift of the absorption maximum induced by increasing the magnetic field. Moreover, we are able to reproduce the current density reversal at the plasma centre for the lowest magnetic field case and its disappearance for higher $B_{0}$. This result is based on the delicate dominance of the second harmonic absorption over the first harmonic absorption at the plasma centre - a somewhat unexpected result. Indeed, as the EBW packet moves along the ray it is propagating away from the second harmonic and approaching the first so that one would expect the opposite result.

In our AMR simulations we have to contend with the loss of about $70 \%$ of the incident power in the first pass due to the low conversion efficiency as well as there being no reliable experimental data on the hot component temperature profile (in our simulations we assumed a homogeneous profile), and the UHR region is completely displaced outside the last closed flux surface where we have only an extrapolated description of the magnetic equilibrium. Yet even under these conditions, our simulations results yield excellent agreement with the WEGA experiments.

While Langmuir probes indicated the existence of a bi-Maxwellian electron distribution, we have shown from our simulations that the hot electron component is vital to reproduce the experimental off-axis absorption and current reversal. Indeed, our simulation results on EBW propagation and absorption are consistent with experimental WEGA results when the 3D ray-tracing code takes into account a supra-thermal electron distribution. Our ray-tracing results indicate an important synergy between the left and the right antenna lobes. Even though the lobes irradiate the plasma surface in opposite directions, at the plasma centre all the waves are propagating in the same direction along the magnetic field $B_{0}$. This effect explains the large total negative current -10 $\mathrm{A} / \mathrm{kW}$.

The experiments demonstrate that the O-X-EBW mode conversion/heating is not restricted just to high magnetic field fusion devices but it is also applicable to low magnetic field plasma experiments for fundamental plasma physics research and industrial applications. 


\section{Acknowledgments}

The work was partly supported by the grant no. 202/08/0419 of Czech Science Foundation, MSMT-7G09042, EFDA, EURATOM, U.S. Department of Energy and and by AS CR project AV0Z-20430508. Permission to publish is granted under Permission to Publish Framework Agreement concluded between Euratom, Institute of Plasma Physics, AS CR, v. v. i., and IOP Publishing Limited.

\section{References}

[1] Otte M et al 2008 AIP Conference Proceedings 9933

[2] Hirshman S P and Vanrij W I 1986 Computer Physics Communications 43143

[3] Podoba Y Y, Laqua H P, Warr G B, Schubert M, Otte M, Marsen S, Wagner F and Holzhauer E 2007 Physical Review Letters $\mathbf{9 8} 255003$

[4] Horvath K, Lingertat J, Otte M and Wagner F 2006 Plasma Physics and Controlled Fusion 48 315

[5] Preinhaelter J and Kopecký V 1973 J. Plas. Phys. 101

[6] Preinhaelter J, Urban J, Laqua H P, Podoba Y, Vahala L and Vahala G 2007 AIP Conference Proceedings 933343

[7] Decker J and Ram A K 2006 Physics of Plasmas 13112503

[8] Ginzburg V L and Rukhadze A A 1975 Volny v magnitoaktivnoi plazme (Moskva: Nauka)

[9] Fried B D and Contte S D 1961 Plasma Dispersion Function (New York: Academic Press)

[10] IAEA AMDIS ALADDIN Database http://www-amdis.iaea.org/ALADDIN

[11] Hayashi M 2003 Bibliography of Electron and Photon Cross Sections with Atoms and Molecules Published in the 20th Century - Argon (Toki: National Institute For Fusion Science)

[12] Bornatici M, Cano R, Debarbieri O and Engelmann F 1983 Nuclear Fusion 231153

[13] Bonoli P T and Englade R C 1986 Physics of Fluids 292937

[14] Fisch N J and Boozer A H 1980 Physical Review Letters 45720

[15] Hansen F R, Lynov J P and Michelsen P 1985 Plasma Physics and Controlled Fusion 271077

[16] Urban J, Preinhaelter J, Diem S J, Laqua H P, Pavlo P, Shevchenko V, Taylor G, Vahala G, Vahala L and Valovič M 2009 Journal of Plasma and Fusion Research SERIES 81153

[17] Urban J and Preinhaelter J 2006 J. Plas. Phys. 721041

[18] Preinhaelter J, Irzak M A, Tregubova E, Vahala L, Vahala G and Zacek F 2001 AIP Proceedings 595326

[19] Piliya A D, Popov A Y and Tregubova E N 2005 Plasma Physics and Controlled Fusion 47379 